\title{
Nonlocal thin films in calculations of the Casimir force
}

\author{
R. Esquivel-Sirvent* \\ Instituto de Fisica, REGINA, Universidad Nacional Autónoma de México, Apartado Postal 20-364, DF 01000 México, Mexico \\ V. B. Svetovoy ${ }^{\dagger}$ \\ MESA + Research Institute, University of Twente, P.O. 217, 7500 AE Enschede, The Netherlands
}

(Received 31 March 2005; published 21 July 2005)

\begin{abstract}
The Casimir force is calculated between plates with thin metallic coating. Thin films are described with spatially dispersive (nonlocal) dielectric functions. For thin films the nonlocal effects are more relevant than for half-spaces. However, it is shown that even for film thickness smaller than the mean free path for electrons, the difference between local and nonlocal calculations of the Casimir force is of the order of a few tenths of a percent. Thus the local description of thin metallic films is adequate within the current experimental precision and range of separations.
\end{abstract}

DOI: 10.1103/PhysRevB.72.045443

PACS number(s): 42.50.Lc, 12.20.Ds, 78.20.-e

\section{INTRODUCTION}

The Casimir force between uncharged metallic plates (also see reviews ${ }^{2-7}$ ) attracted considerable attention in the past years. The force was measured in a number of experiments with high precision using different techniques and geometric configurations. ${ }^{8-16}$ On the other hand, the potential applications of the force in micro- and nanomechanics is still largely unexplored. Actuation and nonlinear behavior of a mechanical oscillator with the Casimir force were demonstrated ${ }^{13}$ and the importance of the force in adhesion and stiction has also been discussed. ${ }^{17-19}$ Due to technological reasons thin coating layers or multilayered structures are often in use in micromechanical devices. The main question to be addressed in this paper is how important are the nonlocal effects when the film thickness is smaller than the mean-free path of the electrons.

For the first time the problem of a thin metallic layer on top of another metal appeared in connection with the first atomic force microscope (AFM) experiments. ${ }^{9,10}$ In these experiments a relatively thick Al layer was covered with $\mathrm{Au} / \mathrm{Pd}$ film of $20 \mathrm{~nm}^{9}$ or $8 \mathrm{~nm}^{10}$ thick to prevent aluminum oxidation. Because the film was thin enough to be transparent for the light with a characteristic frequency $\omega_{c h}=c / 2 a$, where $a$ is the distance between the bodies, it was concluded that the $\mathrm{Au} / \mathrm{Pd}$ layer did not influence the force. ${ }^{20}$ In actual calculations $^{21}$ the thin $\mathrm{Au} / \mathrm{Pd}$ film was changed by a vacuum. This approach was criticized ${ }^{22}$ on the basis that according to the Lifshitz formula ${ }^{23,24}$ the force depends on the dielectric function $\varepsilon(i \zeta)$ at imaginary frequencies $\omega=i \zeta$. The Kramers-Kronig relation shows that at $\zeta_{c h}=c / 2 a$ low real frequencies $\omega \ll c / 2 a$ give a significant contribution to $\varepsilon\left(i \zeta_{c h}\right)$. At low frequencies the $\mathrm{Au} / \mathrm{Pd}$ film is not transparent, and it should be taken into account. It was demonstrated that, indeed, even an $8 \mathrm{~nm}$ thick film gave a significant contribution to the force. The calculation in Ref. 25 supported this conclusion, but the authors speculated that nonlocal effects due to the small thickness of the film (smaller than the meanfree path for electrons) allowed one to consider the film transparent.
The question arose again in connection with a recent experiment, ${ }^{26}$ where the force was measured between a plate and sphere covered with a 10 or $200 \mathrm{~nm}$ Pd film. For a thin film, the expected reduction of the force was clearly observed. It was indicated that spatial dispersion might be important for a calculation of the force in the case of a thin film. ${ }^{27}$ Also, Boström and Sernelius ${ }^{28}$ pointed out the need of detailed studies of nonlocal effects, while studying the retarded van der Waals force between thin metallic films within a local approximation.

There have been several works dealing with the problem of nonlocality in the Casimir force between half-spaces. $\mathrm{Katz}^{29}$ was the first to point out the need of a quantitative study and in his work only a rough estimate of how spatial dispersion affected dispersive forces was given. Heindricks ${ }^{30}$ was able to derive the Lifshitz formula in an approximate way to include nonlocal effects. Similarly, Dubrava using a phenomenological approach described the Casimir attraction between thin films. ${ }^{31}$ More recently, based on the formalism of nonlocal optics, the effects for thick metallic layers have been considered. Propagation of bulk plasmons $\mathrm{s}^{32-34}$ and electromagnetic response in the region of anomalous dispersion ${ }^{35}$ were taken into account, showing that the spatial dispersion does not contribute significantly to the Casimir force. The method developed in Ref. 35 is very general and can be used for the analysis of all nonlocal effects, including those arising in thin films.

\section{FORMALISM}

The Casimir force between two plates separated by a vacuum gap $a$ at a temperature $T$ is given by the Lifshitz formula. ${ }^{23,24}$ The force is expressed via the reflection coefficients $R_{1}$ and $R_{2}$ of the plates 1 and 2, respectively, in the following way:

$$
\begin{aligned}
F_{p p}(a)= & -\frac{k_{B} T}{\pi} \sum_{n=0}^{\infty}, \int_{0}^{\infty} d q q k_{0}\left[\left(R_{1 s}^{-1} R_{2 s}^{-1} \exp \left(2 a k_{0}\right)-1\right)^{-1}\right. \\
& \left.+\left(R_{1 p}^{-1} R_{2 p}^{-1} \exp \left(2 a k_{0}\right)-1\right)^{-1}\right],
\end{aligned}
$$

where subscripts $s$ and $p$ denote the polarization states, $\mathbf{q}$ is 
the wave vector along the plates, $q=|\mathbf{q}|$, and $k_{0}$ is the normal component of the wave vector, defined as

$$
k_{0}=\sqrt{\zeta_{n}^{2} / c^{2}+q^{2}} .
$$

In Eq. (1) the sum is calculated over the Matsubara frequencies,

$$
\zeta_{n}=\frac{2 \pi k_{B} T}{\hbar} n
$$

The reflection coefficients $R_{1}$ and $R_{2}$ are different for $s$ and $p$ polarizations and are functions of $q$ and imaginary frequencies $\zeta_{n}$. They comprise material properties of the plates and for this reason we start our analysis from the reflection coefficients.

\section{A. Local case}

To set our notation, we first study briefly the known local case, when the optical response depends only on frequency. We start the analysis from a thin film of thickness $h$ on a substrate. It will be assumed here that the film is continuous.

For a film on an infinitely thick substrate, the problem is rather simple. The Maxwell equations are solved with the boundary conditions that are the continuity of the tangential components of electric and magnetic fields on both boundaries of the film. The problem can be solved at real frequencies and then analytically continued to the imaginary axis. In the local limit the film and substrate are described by their local dielectric functions, which will be denoted as $\varepsilon_{1}(\omega)$ and $\varepsilon_{2}(\omega)$. In general, the indexes marking the layers will increase from top to bottom of the plate. The dielectric function of the vacuum will be taken as $\varepsilon_{0}(\omega)=1$. The reflection coefficients in our case are well known in optics. At imaginary frequencies they $\operatorname{are}^{36}$

$$
R=\frac{r_{01}-r_{21} \exp \left(-2 k_{1} h\right)}{1-r_{01} r_{21} \exp \left(-2 k_{1} h\right)},
$$

where $r_{m l}$ are the reflection coefficients from the boundary between media $l$ and $m$. These coefficients depend on the polarization, $s$ or $p$, and are defined as

$$
r_{m l}^{s}=\frac{k_{m}-k_{l}}{k_{m}+k_{l}}, \quad r_{m l}^{p}=\frac{\varepsilon_{l} k_{m}-\varepsilon_{m} k_{l}}{\varepsilon_{l} k_{m}+\varepsilon_{m} k_{l}},
$$

where $k_{m}$ is the normal component of the wave vector in the medium $m$ :

$$
k_{m}=\sqrt{\varepsilon_{m}(i \zeta) \frac{\zeta^{2}}{c^{2}}+q^{2}}
$$

It is easy to check that for $h \rightarrow \infty$ (thick film) $R \rightarrow r_{01}$ and in the opposite limit $h \rightarrow 0$ the reflection coefficient coincides with that for the substrate: $R \rightarrow r_{02}$.

To understand the variation of the Casimir force with the film thickness, we first study the behavior of the reflection coefficients. For a qualitative analysis it will be assumed that a metal, film, or substrate, can be described with the Drude dielectric function,

$$
\varepsilon(i \zeta)=1+\frac{\omega_{p}^{2}}{\zeta\left(\zeta+\omega_{\tau}\right)},
$$

where $\omega_{p}$ and $\omega_{\tau}$ are the Drude parameters that are different for each layer. For thin films, $\omega_{\tau}$ is a function of the film thickness. This dependence appears because, in addition to the internal scattering processes, for thin films, scattering from the surfaces is important. These processes are independent of each other and the relaxation time in the Drude model is $\omega_{\tau}=\omega_{\tau}^{\text {bulk }}+\omega_{\tau}^{\text {surf }}(h)$. This effect becomes important when the thickness is smaller than the mean-free path for electron. The dependence on $h$ of $\omega_{\tau}^{\text {surf }}$ is explained by the Fuchs-Sondheimer theory. ${ }^{37,38}$ When $h$ is much smaller than the mean-free path, this dependence is given by

$$
\omega_{\tau}^{\operatorname{surf}}(h)=\frac{3}{8}(1-p) \frac{v_{F}}{h},
$$

where $v_{F}$ is the Fermi velocity and an electron has probability $p$ of being specularly reflected from the surface. As one can see from Eq. (8), only diffusely reflected electrons contribute to $\omega_{\tau}^{\text {surf }}(h)$. Experimental results concerning the specularity are far from unique. Very different values of $p$ in the range $0<p<1$ were used to explain the experimental results. ${ }^{39}$ In this paper we investigate the nonlocal effects for specular reflection of electrons on the surface and do not include in the consideration $h$ dependence of the relaxation frequency. But in any case our results are not very sensitive to the exact value of $\omega_{\tau}$.

Consider first the system consisting of $\mathrm{SiO}_{2}$ substrate with $\varepsilon_{2}=4$ and $\mathrm{Au}$ film on top of it with the parameters $\omega_{p}$ $=9.0 \mathrm{eV}, \omega_{\tau}=0.035 \mathrm{eV} .{ }^{40}$ It is convenient to introduce dimensionless variables and parameters as follows:

$$
\Omega=\frac{\zeta}{\omega_{p}}, \quad Q=\frac{c q}{\omega_{p}}, \quad \gamma=\frac{\omega_{\tau}}{\omega_{p}}, \quad H=\frac{\omega_{p} h}{c} .
$$

The reflection coefficient for $s$ polarization as a function of the dimensionless frequency $\Omega$ is shown in Fig. 1. The dashed curve corresponds to semi-infinite metal $h \rightarrow \infty$. It was calculated with $Q=0.1$. This value is taken for the characteristic wave number $q \sim 1 / 2 a$ at $a \sim 100 \mathrm{~nm}$. The solid lines marked as 1,2, and 3 correspond to the dimensionless thickness $H=0.3,1$, and 3 , respectively. Note that $H=1$ gives the film thickness $h$ equal to the penetration depth $\delta=c / \omega_{p}$ $\approx 22 \mathrm{~nm}(\mathrm{Au})$. One can see that $R_{s}$ decreases fast with the thickness. When $Q$ increases, the film also becomes more transparent for $s$ polarization. The other distinctive feature is that $R_{s}$ is going to zero in the limit $\Omega \rightarrow 0$. In this limit the $s$-polarized field degenerates to pure magnetic field, which penetrate freely via the metallic film.

The reflection coefficient for $p$ polarization shows a different behavior, as one can see in Fig. 2. The dashed line represents the thick film and the solid lines marked as 1 and 2 correspond to $H=1$ and 0.1 , respectively. The variation of $R_{p}$ with the film thickness is not very significant. The reason for this is the effective screening of the $E_{z}$ component, even by a very thin metallic layer. An important conclusion can be drawn from this simple fact. The film thickness affects 


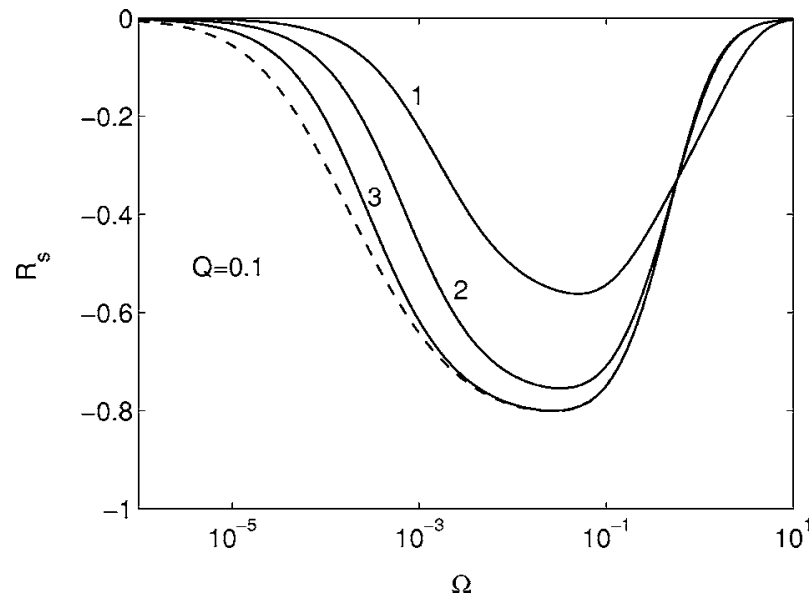

FIG. 1. Reflection coefficient for $s$ polarization as a function of $\Omega$ in the local case (metal film on the dielectric substrate). All the results are presented for $Q=0.1$. An infinitely thick film is given by the dashed line. The curves 1,2 , and 3 correspond to the dimensionless thickness $H=0.3,1$, and 3 , respectively.

mostly the contribution of $s$ polarization, but the part of the force connected with $p$ polarization is changed weakly in the local case.

Consider now the effect of a thin film on top of a thick metallic layer. It will be assumed that both metals can be described by the Drude dielectric functions $\varepsilon_{1}(i \zeta)$ and $\varepsilon_{2}(i \zeta)$ that differ from each other only by the values of parameters $\omega_{i p}$ and $\omega_{i \tau}(i=1,2)$. In this case, the reflection coefficients will be in between the lines describing metal $1(h \rightarrow \infty)$ or metal $2(h \rightarrow 0)$. In Fig. 3 we present the case when the top layer is a better reflector than the bottom one. The dotted line gives $r_{02}$ and the dashed line represents $r_{01}$. The results for the film with thickness $H=1$ and 0.1 are marked as 1 and 2, respectively. In our calculations, the ratios $\omega_{1 p} / \omega_{2 p}=2$ and $\omega_{1 \tau} / \omega_{2 \tau}=1$ were used, and dimensionless parameters (9) were defined relative to the parameters of the top layer 1 . The relaxation frequencies, $\omega_{i \tau}$, influences mostly the low-

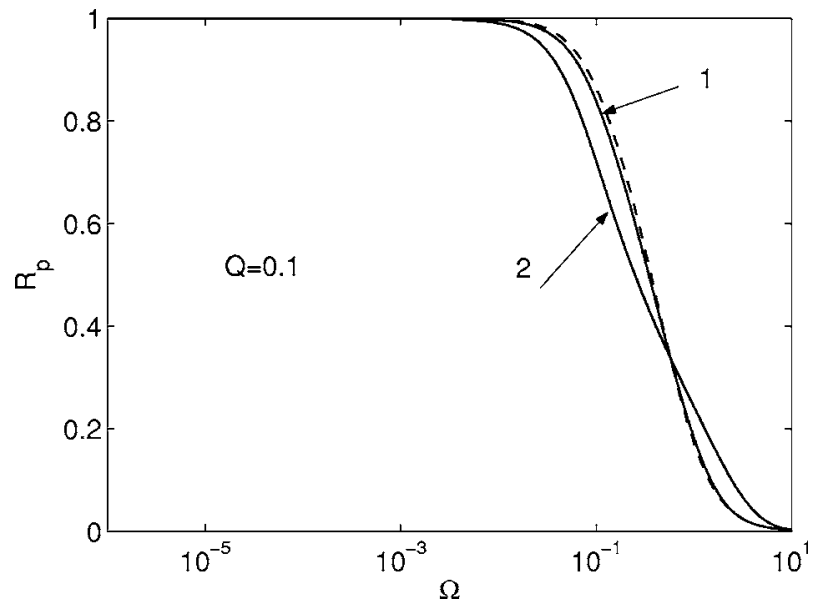

FIG. 2. Reflection coefficient for $p$ polarization as a function of $\Omega$ in the local case (metal film on the dielectric substrate). Infinitely thick layer is shown by the dashed line. The solid lines marked as 1 and 2 correspond to $H=1$ and 0.1 , respectively.

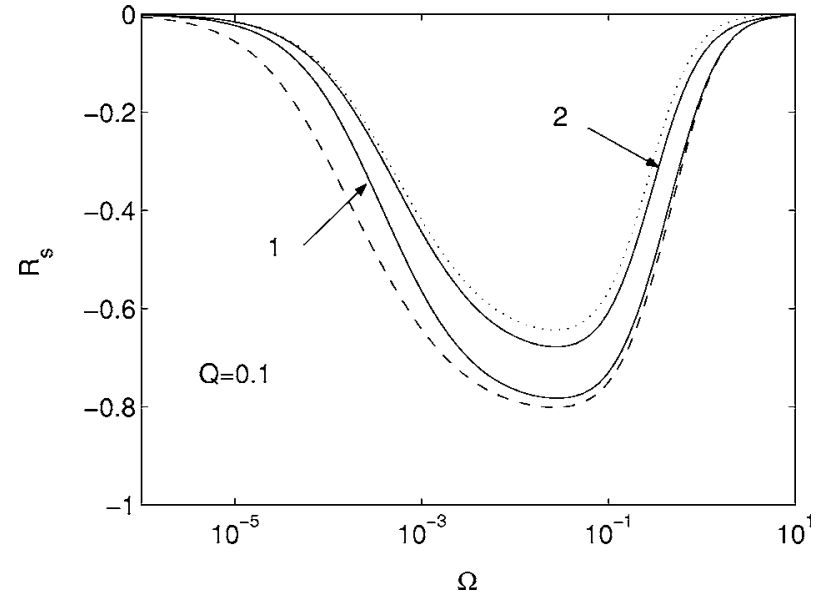

FIG. 3. Reflection coefficient for $s$ polarization as a function of $\Omega$ in the local case (metal film on the metallic substrate). The dotted line represents the substrate, $H=0$, the dashed line represents the thick top layer, $H \rightarrow \infty$. The curves 1 and 2 correspond to $H=1$ and $H=0.1$, respectively.

frequency behavior of $R_{s}$. They are not very important for the Casimir force because the main contribution in the force comes from the imaginary frequencies $\Omega \sim c / 2 a \omega_{p} \gg \gamma$, where $\omega_{\tau}$ does not play a significant role. The reflection coefficient, $R_{p}$, for $p$ polarization is shown in Fig. 4 . The curves 1 and 2 correspond to $H=1$ and $H=0.1$, respectively. Again, one can conclude that the top layer is more important for $s$ than for $p$ polarization.

\section{B. Nonlocal case}

For propagating photons, the reflectivity of thin films in the nonlocal case has been analyzed in Ref. 41. It was assumed that electrons are reflected specularly on both boundaries of the film. Let us consider first $s$ polarization. Similar to the case of a semi-infinite metal, ${ }^{42}$ the tangential component of the electric field is considered as even on each boundary:

$$
E_{y}(m h-z)=+E_{y}(m h+z),
$$

where $z$ is the direction normal to the film surface, $m$ is an arbitrary integer, and the plane of incidence was chosen to be

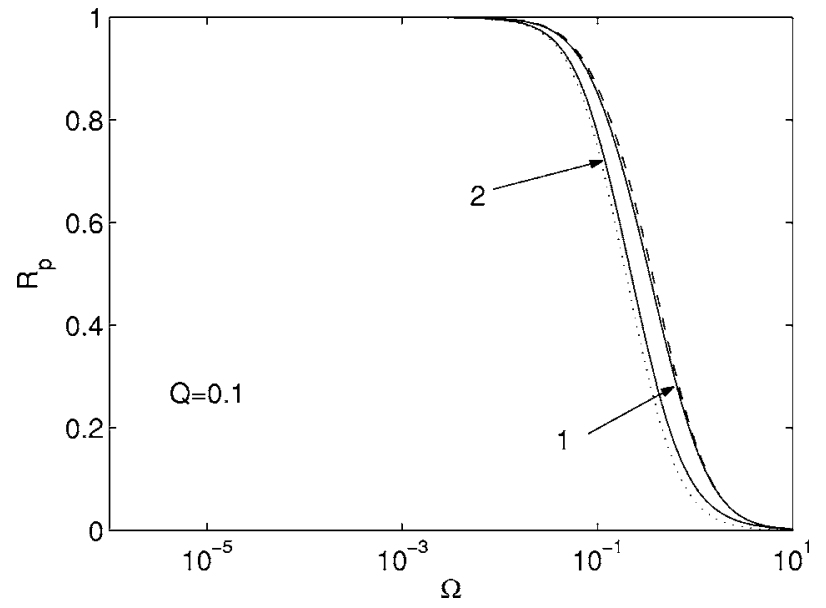

FIG. 4. The same as Fig. 3 but for $p$ polarization 
$x-z$. The Maxwell equations and Eq. (10) demand for the magnetic field on the boundaries the following conditions:

$$
H_{x}(m h-z)=-H_{x}(m h+z), \quad H_{z}(m h-z)=+H_{z}(m h+z) .
$$

Formally the conditions (10) and (11) continue the film of finite thickness to the infinite layer. These conditions mean that the fields can be considered as periodic with period $2 h$, and they can be expanded in a Fourier series.

In the nonlocal case the material is characterized by the impedance instead of local dielectric function. The impedance is defined as the ratio of tangential components of electric and magnetic fields just below the surface. For $s$ and $p$ polarizations, the impedances of metallic film were found in Ref. 41, with the method that is a direct generalization of the method used for the semi-infinite layer. ${ }^{42}$ The film has two surfaces and the impedances one can define on each of them:

$$
Z_{s}=-\left.\frac{E_{y}}{H_{x}}\right|_{z=\delta, h-\delta}, \quad Z_{p}=\left.\frac{E_{x}}{H_{y}}\right|_{z=\delta, h-\delta},
$$

where $\delta \rightarrow 0$. It was noted ${ }^{41}$ that instead of impedances (12) one can use a different couple for each polarization, which can be easy calculated. These new impedances were introduced as the ratio of the fields even or odd relative to the film center $z=h / 2$. Even or odd fields will be marked by the superscripts (1) or (2), respectively. The new impedances,

$$
Z_{s}^{(1,2)}=-\left.\frac{E_{y}^{(1,2)}}{H_{x}^{(1,2)}}\right|_{z=\delta}, \quad Z_{p}^{(1,2)}=\left.\frac{E_{x}^{(1,2)}}{H_{y}^{(1,2)}}\right|_{z=\delta},
$$

are the same on both boundaries of the film because of the symmetry conditions

$$
E_{x, y}^{(1)}(\delta)=E_{x, y}^{(1)}(h-\delta), \quad E_{x, y}^{(2)}(\delta)=-E_{x, y}^{(2)}(h-\delta),
$$

and similarly for the magnetic field.

Explicit expressions for these impedances were found in Ref. 41:

$$
\begin{gathered}
Z_{s}^{(1,2)}=i \frac{2 \omega}{c h} \sum_{n=(\text { odd }, \text { even })} \frac{1}{\frac{\omega^{2}}{c^{2}} \varepsilon_{t}(\omega, k)-\left(\frac{n \pi}{h}\right)^{2}-q^{2}}, \\
Z_{p}^{(1,2)}=i \frac{2 \omega}{c h} \sum_{n=(\text { odd }, \text { even })} \frac{1}{k^{2}}\left[\frac{q^{2}}{\frac{\omega^{2}}{c^{2}} \varepsilon_{l}(\omega, k)}\right. \\
\left.+\frac{\left(\frac{n \pi}{h}\right)^{2}}{\frac{\omega^{2}}{c^{2}} \varepsilon_{t}(\omega, k)-\left(\frac{n \pi}{h}\right)^{2}-q^{2}}\right] .
\end{gathered}
$$

where for even, (1), or odd, (2), fields the sum has to be calculated over $n=2 m+1$ or $n=2 m$, respectively. The transverse dielectric function $\varepsilon_{t}(\omega, k)$ contributes to $Z_{s}$. It describes the response of the material on the electric field transverse to the wave vector $\mathbf{k}$. In case of the $p$-polarization $z$ component of an electric field creates a nonzero charge den- sity in the metal producing the longitudinal field inside of metal. That is why $Z_{p}$ depends also on the longitudinal dielectric function $\varepsilon_{l}(\omega, k)$. In general, these functions are nonlocal, so they depend on both $\omega$ and $k$. The absolute value of the wave vector $\mathbf{k}$ in Eqs. (15) and (16) is

$$
k=\sqrt{\left(\frac{n \pi}{h}\right)^{2}+q^{2}} .
$$

Let us consider now the reflection and transmission coefficients of the film on a substrate. Note that in Ref. 41 only a free-standing film was considered. To find these coefficients, one has to match the tangential components of the electric and magnetic fields outside and inside of the film. We assume for simplicity that the substrate can be described by a local dielectric function or equivalently by local impedances. This assumption is justified by the investigation of nonlocal effects at imaginary frequencies for semi-infinite metals. ${ }^{35} \mathrm{It}$ was demonstrated that in contrast with the real frequencies, the nonlocal effect (anomalous skin effect) brings only a minor influence on the reflection coefficients. Matching the electric field on both sides of the film for $s$ polarization, one gets

$$
\begin{gathered}
E_{y}^{0}\left(1+R_{s}\right)=E_{y}^{(1)}(\delta)+E_{y}^{(2)}(\delta), \\
E_{y}^{0} t_{s} e^{i k_{2} h}=E_{y}^{(1)}(\delta)-E_{y}^{(2)}(\delta),
\end{gathered}
$$

where $E_{y}^{0}$ is the incident field, $t_{s}$ is the transmission coefficient, and the symmetry conditions (14) were taken into account. Similar equations are true for the magnetic field:

$$
\begin{gathered}
H_{x}^{0}\left(1-R_{s}\right)=H_{x}^{(1)}(\delta)+H_{x}^{(2)}(\delta), \\
H_{x}^{0} t_{s} \frac{k_{2}}{k_{0}} e^{i k_{2} h}=-H_{x}^{(1)}(\delta)+H_{x}^{(2)}(\delta) .
\end{gathered}
$$

Equations (18) and (19) can be solved for $R_{s}$ and $t_{s}$, using the impedance definition (13). As a result, the reflection coefficient can be presented in the form

$$
R_{s}=\frac{\left(Z_{s 1}^{(1)}-Z_{s 0}\right)\left(Z_{s 1}^{(2)}+Z_{s 2}\right)+\left(Z_{s 1}^{(2)}-Z_{s 0}\right)\left(Z_{s 1}^{(1)}+Z_{s 2}\right)}{\left(Z_{s 1}^{(1)}+Z_{s 0}\right)\left(Z_{s 1}^{(2)}+Z_{s 2}\right)+\left(Z_{s 1}^{(2)}+Z_{s 0}\right)\left(Z_{s 1}^{(1)}+Z_{s 2}\right)} .
$$

Here we introduced the following notations: $Z_{s 1}^{(1,2)}$ are the nonlocal impedances of the film given by Eq. (13), $Z_{s 2}$ is the local impedance of the substrate defined as

$$
Z_{s 2}=\frac{\omega}{c k_{2}}
$$

and

$$
Z_{s 0}=\frac{\omega}{c k_{0}},
$$

is the "impedance" of the plane wave defined as the ratio of electric and magnetic fields in the wave. The formula (20) for $R_{s}$ cannot be presented in the same form (4) as in the local case. This is because we used the impedances (13) 
instead of that given by Eq. (12). As we will see, both Eqs. (4) and (20) coincide in the local limit.

In the same way, one can find the reflection coefficient for $p$ polarization, $R_{p}$. In this case the equations similar to (18) and (19) with the interchange $x \leftrightarrow y$ will be true, the impedance of the plane wave is defined as

$$
Z_{p 0}=\frac{c k_{0}}{\omega}=\frac{1}{Z_{s 0}},
$$

and the local impedance of the substrate is

$$
Z_{p 2}=\frac{c k_{2}}{\omega \varepsilon_{2}(\omega)} .
$$

The final expression for $R_{p}$ is

$$
R_{p}=-\frac{\left(Z_{p 1}^{(1)}-Z_{p 0}\right)\left(Z_{p 1}^{(2)}+Z_{p 2}\right)+\left(Z_{p 1}^{(2)}-Z_{p 0}\right)\left(Z_{p 1}^{(1)}+Z_{p 2}\right)}{\left(Z_{p 1}^{(1)}+Z_{p 0}\right)\left(Z_{p 1}^{(2)}+Z_{p 2}\right)+\left(Z_{p 1}^{(2)}+Z_{p 0}\right)\left(Z_{p 1}^{(1)}+Z_{p 2}\right)} .
$$

It differs from Eq. (20) only by the general sign and the change $s \rightarrow p$.

If the substrate is changed by vacuum, $Z_{\alpha 2} \rightarrow Z_{\alpha 0}(\alpha$ $=s, p)$, we reproduce the reflection coefficient found in Ref. 41:

$$
R_{\alpha}=\frac{1}{2}\left(r_{\alpha}^{(1)}+r_{\alpha}^{(2)}\right), \quad \alpha=s, p,
$$

where the "partial" reflection coefficients are connected with the impedances by the usual relations

$$
r_{s}^{(1,2)}=-\frac{Z_{s 0}-Z_{s}^{(1,2)}}{Z_{s 0}+Z_{s}^{(1,2)}}, \quad r_{p}^{(1,2)}=\frac{Z_{p 0}-Z_{p}^{(1,2)}}{Z_{p 0}+Z_{p}^{(1,2)}} .
$$

In the local limit both the transverse $\varepsilon_{t}$ and longitudinal $\varepsilon_{l}$ dielectric functions coincide with the local function: $\varepsilon_{t}(\omega, k) \rightarrow \varepsilon_{l}(\omega, k) \rightarrow \varepsilon_{1}(\omega)$. In this case the sums in Eqs. (15) and (16) can be found explicitly. For example, for $s$ polarization one has

$$
Z_{1 s}^{(1), l o c}=-i \frac{\omega}{c k_{1}} \tan \frac{h k_{1}}{2}, \quad Z_{1 s}^{(2), l o c}=i \frac{\omega}{c k_{1}} \cot \frac{h k_{1}}{2} .
$$

Substituting it in Eq. (20), one can check that the reflection coefficient for the local case given by Eq. (4) is reproduced.

All the equations above were written for real frequencies. The transition to imaginary frequencies, which are the main point of our interest, can be done by a simple analytic continuation. To get the nonlocal effects in the reflection coefficients, we have to fix the nonlocal dielectric functions. At imaginary frequencies in the Boltzmann approximation they are given by the relations ${ }^{35}$

$$
\varepsilon_{l}(\Omega, v)=1+\frac{f_{l}(v)}{\Omega(\Omega+\gamma)}, \quad f_{l}(v)=\frac{3}{v^{2}} \cdot \frac{v-\arctan v}{v+\frac{\gamma}{\Omega}(v-\arctan v)},
$$

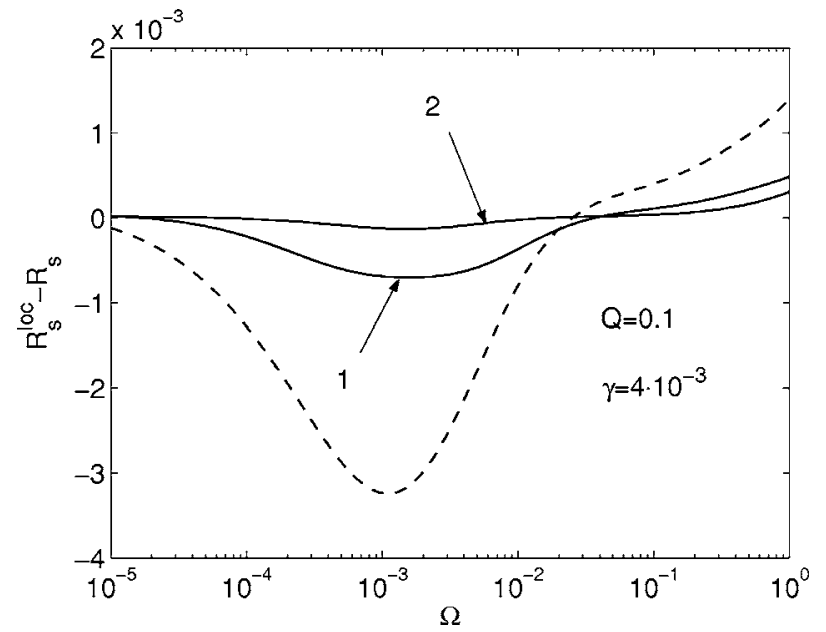

FIG. 5. The difference between the reflection coefficients for $s$ polarization in the local and nonlocal cases (metallic film on the dielectric substrate). The dashed curve was calculated for very thick film. The solid curves are presented for $H=1$ (1) and $H=0.1$ (2).

$$
\begin{gathered}
\varepsilon_{t}(\Omega, v)=1+\frac{f_{t}(v)}{\Omega(\Omega+\gamma)} \\
f_{t}(v)=\frac{3}{2 v^{3}}\left[-v+\left(1+v^{2}\right) \arctan v\right] \\
v=\frac{v_{F}}{c} \frac{\sqrt{\left(\frac{n \pi}{H}\right)^{2}+Q^{2}}}{\Omega+\gamma},
\end{gathered}
$$

where $v_{F}$ is the Fermi velocity. The dimensionless variables (9) have been introduced in Eqs. (29)-(31). In addition, we have neglected in Eqs. (29) and (30) the contribution due to the interband transitions.

The reflection coefficients in the nonlocal case were calculated numerically. In Fig. 5 the difference between local and nonlocal coefficients $R_{s}$ is shown for an Au film on top of the $\mathrm{SiO}_{2}$ substrate. The dashed curve corresponds to a very thick film, $H \rightarrow \infty$. The solid lines marked as 1 and 2 are presented for $H=1$ and 0.1 , respectively. As before, $H=1$ corresponds to the penetration depth of Au $(\delta=22 \mathrm{~nm})$. The thick film clearly demonstrates the anomalous skin effect at $\Omega \sim \gamma$, although the magnitude of the effect is small, as was already noted in Ref. 35. Even this small effect decreases with the film thickness, as the curves 1 and 2 show. The nonlocal effect increases with $Q$ but it is smaller than $1 \%$, even for $Q=1$. It should be noted that the Boltzmann approximation is good while $\Omega<1$, but when $\Omega$ is approaching 1 the reflection coefficient itself becomes small, and there is no sense to keep the nonlocal correction in this range. A similar result was found for the film on top of a metallic substrate. One can conclude that for $s$ polarization the nonlocal effect in the reflection coefficient is very small and can be neglected in calculation of the Casimir force.

The situation for $p$ polarization is shown in Fig. 6 for the film on top of a metallic substrate. As in the local case the 


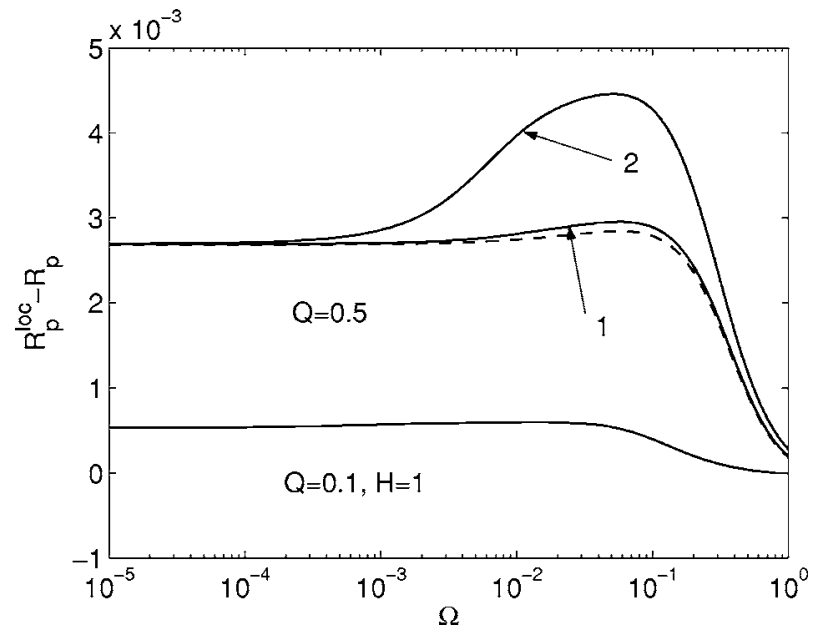

FIG. 6. The difference between the reflection coefficients for $p$ polarization in the local and nonlocal cases (metal film on the metallic substrate). The lower line is given for $Q=0.1, H=1$. The upper series is for $Q=0.5$. The dashed curve presents a very thick film. The solid curves are given for $H=1$ (1) and $H=0.1$ (2).

substrate was chosen to have the plasma frequency 2 times smaller than that for the film. The lower curve corresponds to $Q=0.1$ and $H=1$. The upper series of curves is given for $Q$ $=0.5$. As one can see, the nonlocal effect manifests itself in a wider frequency range and does not disappear, even for zero frequency. The latter is the result of Thomas-Fermi screening, as was explained in Ref. 35. The effect is still small, but the nonlocal contribution in the Casimir force will be larger than that for $s$ polarization. This is because the nonlocal effect is the largest at frequencies that give the main contribution in the Casimir force.

\section{EFFECTS OF SPATIAL DISPERSION ON THE CASIMIR FORCE}

To quantify the effect of spatial dispersion on the Casimir force, we calculate the percent difference between the local case and nonlocal case $\left[\Delta \%=\left|\left(F_{\text {local }}-F_{\text {nonlocal }}\right) / F_{\text {local }}\right|\right]$, as a function of separation.

First we consider the case of free-standing metallic films. The system is similar to that considered by Boström and Sernelius. ${ }^{28}$ The percent difference $\Delta \%$ as a function of separation is presented in Fig. 7, for three different thicknesses. The results for the thick film $h=100 \mathrm{~nm}$ coincides with the results obtained for half-spaces in our previous work. ${ }^{35}$ As the thickness decreases, the nonlocal effects become more relevant. Thin films have a more complicated nonlocal response than half-spaces. For $p$-polarized waves, surface plasmons on each side of the film can interfere, ${ }^{43}$ creating standing waves that will increase the electromagnetic absorption of the field that will decrease the Casimir force. These resonance conditions are evident from Eq. (17), where $k_{z}$ $=n \pi / L$.

The force is not affected significantly when the thin films are on substrates. In Fig. 8 we have plotted the percent difference between two thin Au films, each deposited on a dielectric substrate. Again, we assumed $\epsilon=4$ for the dielectric,

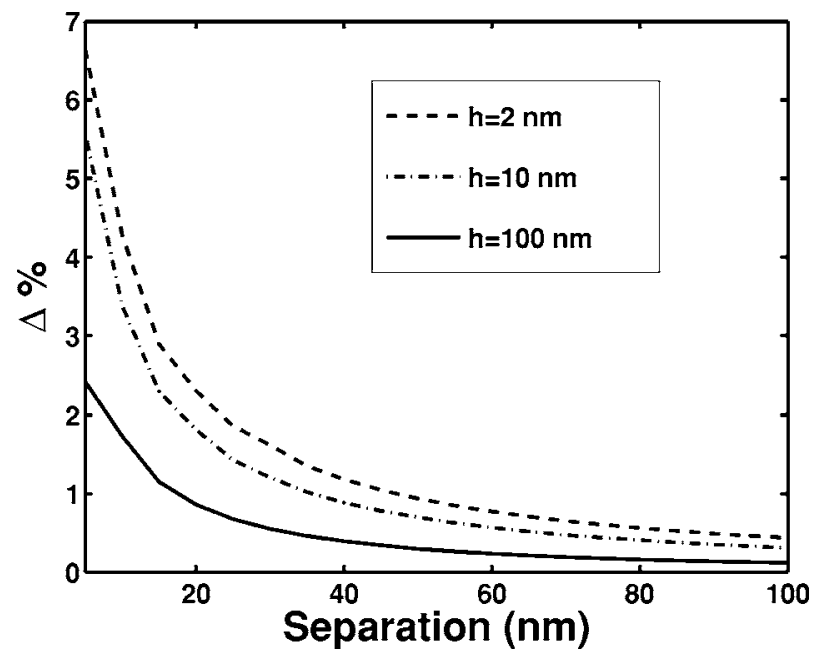

FIG. 7. Percent difference $\Delta \%$ between the local and nonlocal Casimir force between Au free-standing films. The values for the thick film coincide with those obtained for half-spaces (Ref. 35).

just as an illustrative example of the effect of substrate. The substrates reduce slightly the value of $\Delta \%$ for both curves shown, with the obvious limit that when the substrate has the same dielectric function as the film, we recover the results for the force between half-spaces. This means that the effect of the substrate is to allow energy transfer out of the thin film into the substrate.

The difference between the local and nonlocal cases can be reduced in a system consisting of Au half-space and $\mathrm{Au}-$ coated substrate. Again, we took a dielectric $(\epsilon=4)$. The effect of spatial dispersion reduces significantly as compared to the cases treated in Figs. 7 and 8. This shows that the most important part of the spatial dispersion effect comes from the thin films. If in current experiments the separation can go down to $50 \mathrm{~nm}$, in the system shown in Fig. 9, the nonlocal correction is of the order of $0.34 \%$.

The result holds for different substrates. This is shown in Table I, where we presented the percent difference between the local and nonlocal forces for the Au film deposited on different substrates. All data are given for a separation of $50 \mathrm{~nm}$. As before, $\omega_{p 1} / \omega_{p 2}$ is the ratio of the Au plasma

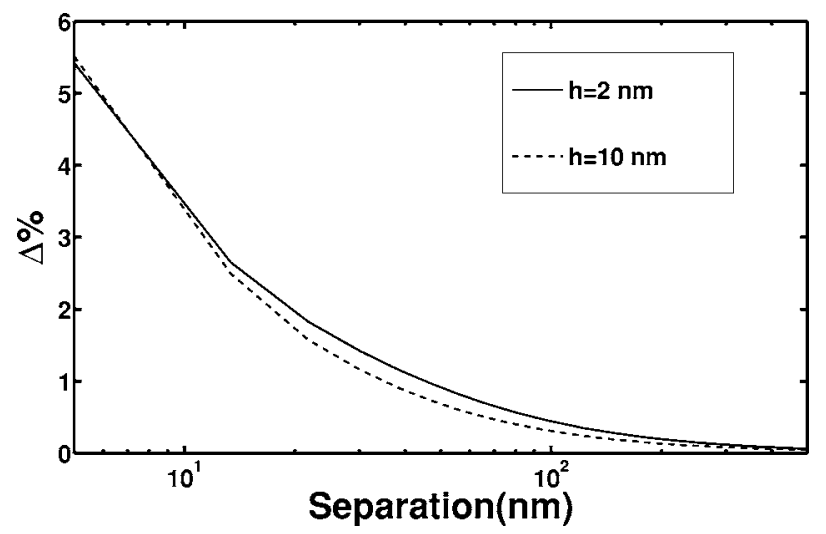

FIG. 8. Percent difference $\Delta \%$ between the local and nonlocal Casimir force between two Au films deposited on a dielectric substrate. 


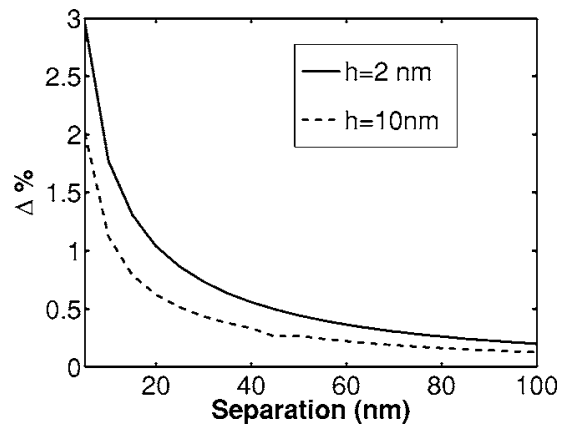

FIG. 9. Percent difference $\Delta \%$ between the local and nonlocal Casimir force between Au half-space and Au-coated dielectric.

frequency to that of the metallic substrate, assuming the damping factor remains the same. The case $\varepsilon=1$ corresponds to the free-standing Au thin film (no substrate).

\section{CONCLUSIONS}

The role of thin metallic coatings in the calculation of Casimir forces has been studied, taking into account spatial dispersion. The description of the nonlocal response of thin films is based on the Kliewer and Fuchs formalism that imposes a symmetrical behavior of the fields inside thin films. The study of the reflectivities shows that the main contribution to the nonlocal effect comes from $p$-polarized light that excites normal modes within the material. At very small separations, the effects can be appreciable, but at best a percent difference of $7 \%$ is found. However, for typical experimental setups and separations the percent difference between
TABLE I. The magnitude of the nonlocal effect for different substrates at a fixed separation of $50 \mathrm{~nm}$ and a film thickness of $2 \mathrm{~nm}$.

\begin{tabular}{cc}
\hline \hline Substrate & $\Delta \%$ \\
Dielectric, $\epsilon=4$ & 0.34 \\
Metal, $\omega_{p 1} / \omega_{p 2}=2$ & 0.37 \\
Metal, $\omega_{p 1} / \omega_{p 2}=0.5$ & 0.44 \\
No substrate, $\epsilon=1$ & 0.44 \\
\hline \hline
\end{tabular}

the local and nonlocal case is of the order of $0.4 \%$, that can be regarded as negligible within current experimental precisions and the local description is good enough. The effect of thin films within a local approximation has been measured recently by Lisanti et al. $^{26}$

Along with the previous works on nonlocal effects between half-spaces, ${ }^{32-35}$ we can generally conclude that these effects will be difficult to detect at the current experimental precision. Our results indicate a decrease in the force due to spatial dispersion. However for half-spaces within a jellium model it has been shown ${ }^{34}$ that the force can increase due to nonlocal effects because of decrease in the separation of the optical surfaces that might not coincide with the physical surface.

\section{ACKNOWLEDGMENTS}

We acknowledge partial support from CONACyT Project 44306 and DGAPA-UNAM IN-101605. We thank W. L. Mochan and C. Villarreal for helpful discusions.
*Electronic address: raul@ fisica.unam.mx

${ }^{\dagger}$ On leave from Yaroslavl University, Yaroslavl, Russia. Electronic address: V.B.Svetovoy@el.utwente.nl

${ }^{1}$ H. B. G. Casimir, Proc. K. Ned. Akad. Wet. 51, 793 (1948).

${ }^{2}$ P. W. Milonni, The Quantum Vacuum (Academic Press, San Diego, 1994).

${ }^{3}$ V. M. Mostepanenko and N. N. Trunov, The Casimir Effect and its Applications (Clarendon Press, Oxford, 1997).

${ }^{4}$ M. Kardar and R. Golestanian, Rev. Mod. Phys. 71, 1233 (1999).

${ }^{5}$ K. A. Milton, The Casimir Effect (World Scientific, Singapore, 2001).

${ }^{6}$ M. Bordag, U. Mohideen, and V. M. Mostepanenko, Phys. Rep. 353, 1 (2001).

${ }^{7}$ K. A. Milton, J. Phys. A 37, R209 (2004).

${ }^{8}$ S. K. Lamoreaux, Phys. Rev. Lett. 78, 5 (1997); 81, 5475 (1998).

${ }^{9}$ U. Mohideen and A. Roy, Phys. Rev. Lett. 81, 4549 (1998).

${ }^{10}$ A. Roy, C.-Y. Lin, and U. Mohideen, Phys. Rev. D 60, 111101(R) (1999).

${ }^{11}$ B. W. Harris, F. Chen, and U. Mohideen, Phys. Rev. A 62, 052109 (2000).

${ }^{12}$ T. Ederth, Phys. Rev. A 62, 062104 (2000).

${ }^{13}$ H. B. Chan, V. A. Aksyuk, R. N. Kleiman, D. J. Bishop, and F. Capasso, Science 291, 1941 (2001); Phys. Rev. Lett. 87,
211801 (2001).

${ }^{14}$ G. Bressi, G. Carugno, R. Onofrio, and G. Ruoso, Phys. Rev. Lett. 88, 041804 (2002).

${ }^{15}$ R. S. Decca, D. López, E. Fischbach, and D. E. Krause, Phys. Rev. Lett. 91, 050402 (2003).

${ }^{16}$ R. S. Decca, E. Fischbach, G. L. Klimchitskaya, D. E. Krause, D. López, and V. M. Mostepanenko, Phys. Rev. D 68, 116003 (2003).

${ }^{17}$ E. Buks and M. L. Roukes, Phys. Rev. B 63, 033402 (2001).

${ }^{18}$ R. W. Johnstone and M. Parameswaran, J. Micromech. Microeng. 12, 855 (2002).

${ }^{19}$ Y.-P. Zhao, L. S. Wang, and T. X. Yu, J. Adhes. Sci. Technol. 17, 519 (2003).

${ }^{20}$ For most of the experiments, the force is measured between a large sphere and a plane, $a$ being the closest distance between the sphere's surface and the plane. In the rest of this work we will refer to $a$ also as the separation between two parallel planes.

${ }^{21}$ G. L. Klimchitskaya, A. Roy, U. Mohideen, and V. M. Mostepanenko, Phys. Rev. A 60, 3487 (1999).

${ }^{22}$ V. B. Svetovoy and M. V. Lokhanin, Mod. Phys. Lett. A 15, 1013 (2000).

${ }^{23}$ E. M. Lifshitz, Zh. Eksp. Teor. Fiz. 29, 94 (1956) [Sov. Phys. JETP 2, 73 (1956)]. 
${ }^{24}$ E. M. Lifshitz and L. P. Pitaevskii, Statistical Physics, Part 2 (Pergamon Press, Oxford, 1980).

${ }^{25}$ G. L. Klimchitskaya, U. Mohideen, and V. M. Mostepanenko, Phys. Rev. A 61, 062107 (2000).

${ }^{26}$ M. Lissanti, D. Iannuzzi, and F. Capasso, quant-ph/0502123v1 2005.

${ }^{27}$ D. Iannuzzi, I. Gelfand, M. Lisanti, and F. Capasso, Proceedings of the Quantum Field Theory Under External Conditions 2003, edited by K. A. Milton (Rinton Press, Princeton, NJ, 2003), p. 11; quant-ph/0312043.

${ }^{28}$ M. Bostrom and B. E. Sernelius, Phys. Rev. B 62, 7523 (2000).

${ }^{29}$ E. I. Katz, Sov. Phys. JETP 46, 109 (1977).

${ }^{30}$ J. Heindricks, Phys. Rev. B 11, 3625 (1975).

${ }^{31}$ V. N. Dubrava and V. A. Yampolskii, Low Temp. Phys. 25, 979 (1999).

${ }^{32}$ R. Esquivel, C. Villarreal, and W. L. Mochan, Phys. Rev. A 68, 052103 (2003).
${ }^{33}$ R. Esquivel, C. Villarreal, and W. L. Mochan, Phys. Rev. A 71, 029904(E) (2005).

${ }^{34}$ A. M. Contreras Reyes, Ph. D. thesis, Universidad de las Américas, 2003 (in Spanish).

${ }^{35}$ R. Esquivel and V. B. Svetovoy, Phys. Rev. A 69, 062102 (2004).

${ }^{36}$ F. Zhou and L. Spruch, Phys. Rev. A 52, 297 (1995).

${ }^{37}$ K. Fuchs, Proc. Cambridge Philos. Soc. 34, 100 (1938).

${ }^{38}$ E. H. Sondheimer, Adv. Phys. 1, 1 (1952).

${ }^{39}$ G. Fischer, H. Hoffmann, and J. Vancea, Phys. Rev. B 22, 6065 (1980).

${ }^{40}$ A. Lambrecht and S. Reynaud, Eur. Phys. J. D 8, 309 (2000).

${ }^{41}$ W. E. Jones, K. L. Kliewer, and R. Fuchs, Phys. Rev. 178, 1201 (1969).

${ }^{42}$ K. L. Kliewer and R. Fuchs, Phys. Rev. 172, 607 (1968).

${ }^{43}$ T. López-Rios, Spatial Dispersion in Solids and Plasmas, edited by P. Halevi (North-Holland, New York, 1992), p. 217. 